\title{
How ill-defined constituents produce well-defined nanoparticles: Effect of polymer dispersity on the uniformity of copolymeric micelles
}

\author{
Sriteja Mantha, ${ }^{1, *}$ Shuanhu Qi, ${ }^{1, \dagger}$ Matthias Barz, ${ }^{2, \ddagger}$ and Friederike Schmid ${ }^{1, \S}$ \\ ${ }^{1}$ Institut für Physik, Johannes Gutenberg Universität Mainz, Staudingerweg 9, 55128 Mainz, Germany \\ ${ }^{2}$ Institut für Organische Chemie, Johannes Gutenberg Universität Mainz, Duesbergweg 10-14, 55128 Mainz, Germany
}

(Received 10 May 2018; revised manuscript received 30 October 2018; published 12 February 2019)

\begin{abstract}
We investigate the effect of polymer length dispersity on the properties of self-assembled micelles in solution by self-consistent field calculations. Polydispersity stabilizes micelles by raising the free energy barriers of micelle formation and dissolution. Most importantly, it significantly reduces the size fluctuations of micelles: Block copolymers of moderate polydispersity form more uniform particles than their monodisperse counterparts. We attribute this to the fact that the packing of the solvophobic monomers in the core can be optimized if the constituent polymers have different length.
\end{abstract}

DOI: 10.1103/PhysRevMaterials.3.026002

\section{INTRODUCTION}

Polymeric nanoparticles are attracting considerable and growing interest because of their potential in materials science [1-3] and in nanomedicine, e.g., in targeted therapeutic or diagnostic systems [4,5]. One attractive way to fabricate such nanoparticles is to exploit the self-assembly of amphiphilic block copolymers in solution. Depending on the lengths and solubilities of the copolymer blocks, they can form a variety of interesting morphologies, such as vesicles, rods, and spherical micelles $[6,7]$.

In the present paper, we consider the micelles, which have a core-shell structure with a solvophobic core and a solvophilic shell exposed to the solvent [8-10]. In principle, the core can be exploited to load the drug molecules, and efficient mechanisms can be devised to enhance bioavailability [11-13]. As long as the drug is stably encapsulated, it is believed that the distribution of drug-loaded polymeric micelles in the body is determined by the size and surface properties of polymeric micelles rather than the properties of the drug molecules [14-16]. Hence developing strategies to control the size distribution of polymeric micelles is important to improve the efficacy of targeted drug delivery techniques.

However, all synthetic polymers, including block copolymers, possess some inherent dispersity in the polymer length due to the nature of polymerization reaction [17-19]. Currently, it is not understood to which extent the polydispersity of block copolymers affects the dispersity of the formed micelles-especially since even monodisperse amphiphiles do not form monodisperse micelles [20]. Polydisperse poly-

\footnotetext{
*smantha@uni-mainz.de

†qishuanhu@ buaa.edu.cn; present address: Key Laboratory of BioInspired Smart Interfacial Science and Technology of Ministry of Education, School of Chemistry, Beihang University, Beijing 100191, China.

*barz@uni-mainz.de

§schmidfr@uni-mainz.de
}

mer systems contain individual polymers with shorter or longer blocks. This provides an entropic advantage in the self-assembly process, since long chains can fill the center of domains without having to stretch, whereas short chains adopt conformations near the interface $[18,19]$. Indeed, experiments on ABA triblock copolymer melts have indicated that polydispersity greatly enhances the stability of selfassembled lamellar structures [21]. Related observations have been made in theoretical studies of polymer brushes: They indicate that monodisperse brushes show multicritical behavior $[22,23]$, which are associated with large anomalous chain fluctuations [24-26] that disappear in polydisperse brushes [22]. Hence it is not a priori clear whether polydispersity will enhance or reduce the size fluctuations of self-assembled micelles.

This question is addressed in the present paper. We use self-consistent field (SCF) theory to study the structures and free energy landscapes of micelles that assemble from polydisperse polymer solutions for varying polydispersity index (Đ) close to the critical micelle concentration (CMC), i.e., the point where micelles just begin to form.

Studies of polydispersity effects on self-assembled nanostructures are still comparatively scarce (for reviews see Refs. $[18,19])$. Most studies have considered polydispersity effects on the self-organization in block copolymer melts $[18,27-41]$. Early theoretical studies $[42,43]$ on micellar solutions have predicted that the CMC decreases with polydispersity [42] and that the polymers in the micelles are on average longer than in the surrounding solution. This was later confirmed by experiments $[44,45]$. The size of vesicles formed by amphiphilic diblock copolymers in solution was reported to decrease with increasing dispersity of the hydrophilic block [46-48]. In contrast, experimental studies on micelles made of amphiphilic triblocks with polydisperse inner (hydrophobic) block have shown that the micelle sizes may increase significantly with $Ð$ (at comparable average chain length) [49], in agreement with theoretical predictions [43], and that micelles may even become slightly oblate for high polydispersities [49]. The latter effect is however small (the aspect ratio is 
$1: 1.4)$, and since we focus on moderate polydispersities in the present work, we will assume that micelles are spherically symmetric.

\section{MODEL AND METHOD}

We study systems of polydisperse amphiphilic diblock copolymers with solvophobic blocks A (chain fraction f) and solvophilic blocks B [chain fraction (1-f)] immersed in a solvent $\mathrm{S}$, in the grand canonical ensemble. Since every copolymer length $N$ defines a separate species, we must introduce separate chemical potentials $\mu_{P}(N)$ for each. They are adjusted such that copolymers in solution are distributed according to a Schulz-Zimm distribution [50] $P_{S Z}(N)$ with average chain length $N_{n}=\sum_{N} N P_{S Z}(N)$ and polydispersity index $Đ=\sum_{N} N^{2} P(N) / N_{n}^{2}$. Note that the chain length distribution in the micelles may differ from $P_{S Z}(N)$.

Polymers are modeled as flexible Gaussian chains [51,52]. The segment interactions are characterized by Flory-Huggins parameters $\chi_{i j} N_{n}(i, j=A, B, S)$ and are chosen similar to previous work [53] as $\chi_{A B} N_{n}=10.0, \chi_{A S} N_{n}=17.4$, and $\chi_{B S} N_{n}=-0.5$. The system is compressible with compressibility (Helfand) parameter $\kappa_{H} N_{n}=100$. The structure and the free energy of spherically symmetric micelles are calculated within the SCF theory $[52,54,55]$. The model equations, model parameters, and further details are given in the Supplemental Material [56]. The micelle free energy $F_{m}$ is obtained from the free energy difference of a system containing a micelle and the corresponding ("bulk") homogeneous system. The micelle radius is defined as the radius where the solvophobic density assumes the value $\Phi_{A}=0.5$. In the following, spatial distances are reported in units of radius of gyration $\left(\bar{R}_{g}\right)$ of ideal Gaussian chains with length $N_{n}\left(\bar{R}_{g}^{2}=\frac{N_{n} b^{2}}{6}\right)$, densities are made dimensionless by dividing them by the bulk segment density $\rho_{0}$, and energies are given in units of $\bar{C} k_{B} T$, where $\bar{C}=\bar{R}_{g}^{3} \rho_{o} / N_{n}$ is the Ginzburg parameter which characterizes the strength of fluctuations in the system [52,57]. In the present paper, we consider block copolymers with $\mathrm{A}$ and $\mathrm{B}$ blocks of equal average molecular weight $(\langle f\rangle=0.5)$, as in the experimental system of Ref. [49].

\section{RESULTS}

\section{A. Micelle structures and size distributions}

We first consider copolymers with fixed block ratio $f=$ 0.5 . We compare systems with same average chain length in the bulk and same micelle free energy $F_{m} / k_{B} T \approx 0$ (where micelles just begin to form) but varying polydispersity index Đ. To fix $F_{m}$, the bulk polymer volume fraction $\bar{\Phi}_{p}$ must be adjusted. We find that $\bar{\Phi}_{P}$ decreases significantly with $Đ$ (Fig. 1, inset), indicating that micelle formation sets in for smaller copolymer concentrations in polydisperse solutions. Moreover, the micelle size increases with $Đ$ (Fig. 1, left). The reason becomes clear when examining the chain length distribution in the micelles (Fig. 1, right). In polydisperse systems, it differs significantly from the chain length distribution in the bulk. The largest chains in solution aggregate first, presumably because they can form aggregates at lower cost of translational entropy, hence micelles become bigger. These
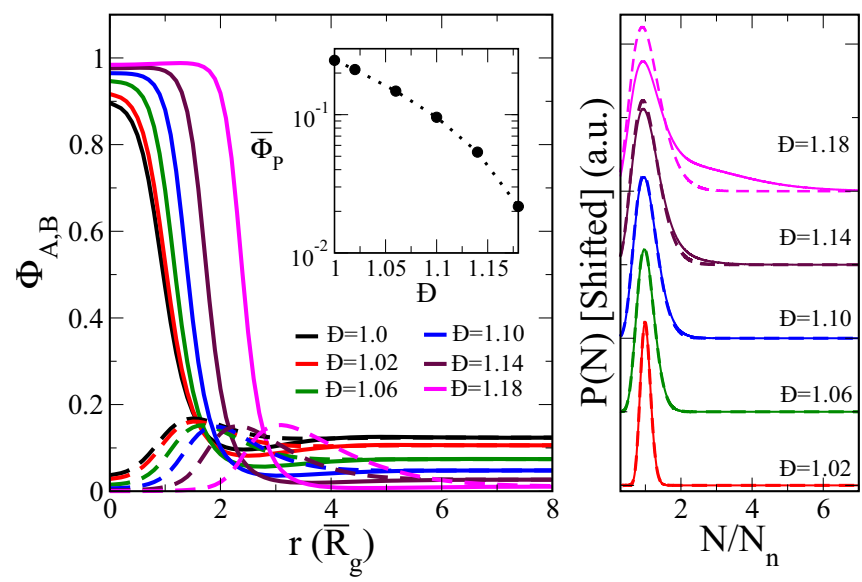

FIG. 1. Left: Spatial density profiles of solvophobic A segments (full lines) and solvophilic B segments (dashed lines) in micelles made of A:B copolymers with $f=0.5, F_{m} / k_{B} T \approx 0$ for different bulk polydispersity indices $\mathrm{D}$ as indicated. Inset shows the corresponding shift of the polymer volume fraction $\bar{\Phi}_{p}$ in the bulk. Right: Chain length distribution of copolymers in the bulk (dashed) and in the micelle (solid) for different values of $Đ$. A constant offset has been added for better visibility.

effects are in agreement with earlier theoretical predictions $[42,43]$ and experimental results $[45,49]$. Similar effects are observed for critical nuclei in polymer mixtures [58].

Next we study the size distribution of micelles. To this end, we determine the constrained free energy $F_{m}(R)$ for micelles of fixed radius $R$ (see the Supplemental Material [56] for technical details). The probability for finding a micelle with size $R$ is then proportional to $\exp \left(-F_{m}(R) / k_{B} T\right)$. The function $F_{m}(R)$ is shown in Fig. 2(left). It starts at $F_{m}(0)=$ 0 , then exhibits a maximum followed by a minimum. The minimum corresponds to the most probable micelle size $R_{m p}$ and coincides with the solution of the unconstrained SCF equations discussed above (Fig. 1). Consistent with Fig. 1, it shifts to the right with increasing polymer polydispersity. The maximum correspond to an unstable micelle state: Micelles of this size may dissolve again. We will refer to it as critical micelle size $R_{m c}$. The height of the maximum gives the free energy barrier for micelle formation, and the free energy difference $\Delta F_{m}=F_{m}\left(R_{m c}\right)-F_{m}\left(R_{m p}\right)$ gives the free energy barrier for micelle dissolution. According to Fig. 2, these barriers increase with increasing polydispersity. Hence polydispersity stabilizes micelles, suggesting that they might also have narrower size distributions.

The micelle size dispersity is characterized by the relative width of the size distribution, $\sigma_{m}=\sqrt{\left\langle R^{2}\right\rangle /\langle R\rangle^{2}-1}$. To calculate $\sigma_{m}$, we fit the SCF results for $F_{m}(R)$ to a fourth order polynomial (see Fig. 2, left) and use that to determine the averages $\left\langle R^{k}\right\rangle=I_{k} / I_{0}$ with $I_{k}=\int_{R_{m}}^{\infty} \mathrm{d} R R^{k} \mathrm{e}^{-F_{m}(R) / k_{B} T}$. In doing so, we must specify a value for the global prefactor $\bar{C}$ in $F_{m}(R)$ (see Fig. 2, left). The Ginzburg $\bar{C}$ is related to a complementary parameter called the invariant polymerization index $(\bar{N})$ as, $\bar{N} \propto \bar{C}^{2}$, where $\bar{N}=N_{n} \rho_{0}^{2} b^{6}$. Typical values for $\bar{N}$ in experimental systems are [59-62] $\bar{N} \simeq 200-20000$, which corresponds to $\bar{C} \simeq 1-10$. Figure 2 (right) shows our results for $\sigma_{m}$ as a function of $Đ$ for three different choices 


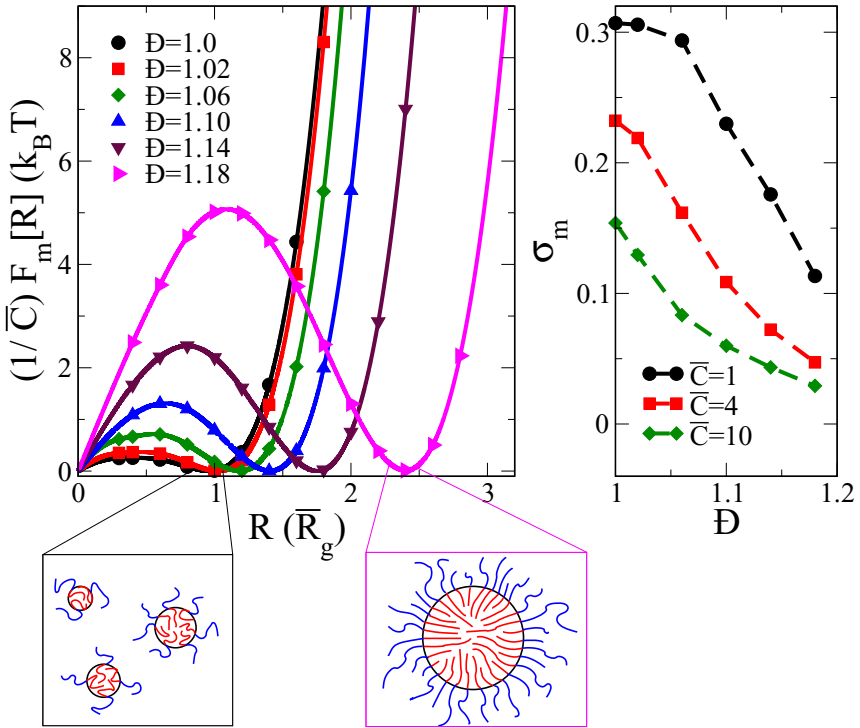

FIG. 2. Left: Micelle free energy as a function of micelle radius for the same systems as in Fig. 1. Symbols show SCF results, solid lines a fit to the polynomial $\sum_{n=1}^{4} a_{n} x^{n}$. Right: Corresponding micelle size dispersity $\sigma_{m}$ as a function of copolymer polydispersity index $Ð$ for different values of the scaling parameter $\bar{C}$. Cartoons illustrate the proposed stabilizing mechanism: Polydisperse solvophobic blocks can pack more efficiently in the core of the micelle.
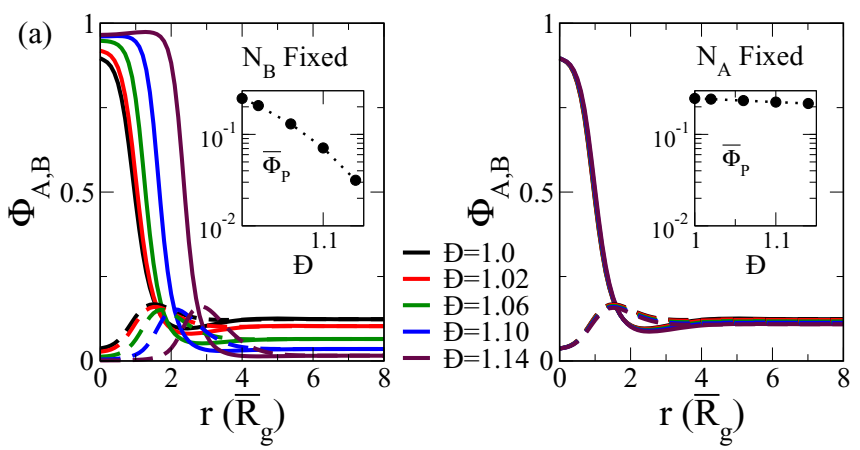

(b)
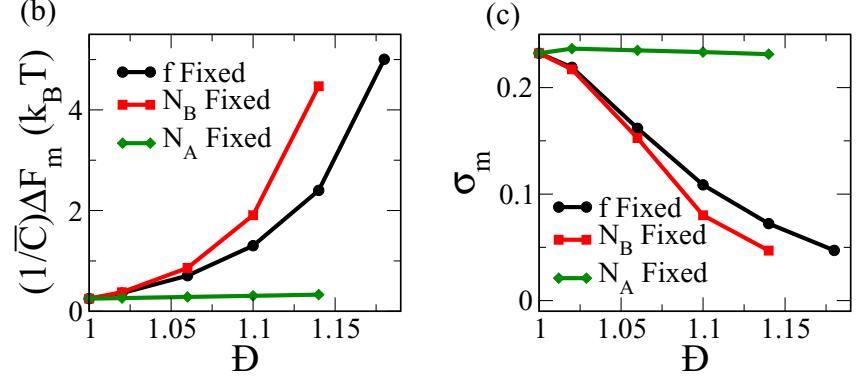

FIG. 3. (a) Density profiles of A and B segments (full/dashed lines) for micelles with micelle free energy $\Delta F_{m}=0$ in systems where only the solvophobic (left) or solvophilic (right) chain block is polydisperse. The inset shows the corresponding bulk polymer volume fractions $\bar{\Phi}_{P}$. (b) Height $\Delta F_{m}$ of the free energy barrier for micelle dissolution. (c) Width $\sigma_{m}$ of micelle size distribution at $\bar{C}=4$ vs $Đ$ for all systems considered in the present work. Here $Đ$ refers to the dispersity in the corresponding polydisperse part of the chain (A or B or total).
$\bar{C}=1,4$, and 10 . In all cases, the micelle size dispersity $\sigma_{m}$ decreases with increasing polymer dispersity Đ. Thus we find that polymer dispersity not only stabilizes micelles but also reduces their size dispersity. This is the main result of the present paper.

\section{B. Other copolymer architectures}

To further investigate this phenomenon, we study two other classes of systems where the copolymer blocks still have equal length on average $(\langle f\rangle=0.5)$ but are now varied independently: In the first system, this solvophobic block is polydisperse with $\left\langle N_{A}\right\rangle / N_{n}=0.5$ and the length of the solvophilic block is kept fixed at $N_{B} / N_{n}=0.5$. In the second system, the solvophobic block is fixed at $N_{A} / N_{n}=0.5$ and the length of the solvopholic block fluctuates with $\left\langle N_{B}\right\rangle / N_{n}=$ 0.5 .

The main results of the SCF calculations are compiled in Fig. 3. If only the solvophobic block is polydisperse and the solvophilic block is kept monodisperse, the effect of polydispersity on the micelle size [Fig. 3(a), left], the chain length distribution (Fig. S2 in the Supplemental Material [56]), the height of the free energy barrier $\Delta F_{m}$ [Fig. 3(b)], and the micelle dispersity [Fig. 3(c)] is even stronger than before. In contrast, if the solvophobic block is monodisperse, polydispersity of the solvophilic block has almost no influence on the micelle size [Fig. 3(a), right] and the other micelle characteristics. Hence the micelle structure and size distribution in the solution is primarily determined by the dispersity of the solvophobic chain block. These results suggest that the main effect of polydispersity is to enhance the packing efficiency inside the hydrophobic core.

\section{Free energy analysis}

To test this hypothesis, we separate the different contributions to the micelle free energy according to $F_{m}=\Delta U+$ $W_{\text {chem }}-T \Delta S$, where $\Delta U$ and $\Delta S$ are the interaction energy and entropy in the micelle relative to the bulk, and $W_{\text {chem }}=$ $-\mu_{s} \Delta n_{s}-\sum_{N} \mu_{p}(N) \Delta n_{P}(N)$ refers to the chemical work required for bringing polymer into the system and moving solvent out ( $\Delta n_{x}$ is the excess number of molecules of type $X$ in the micelle). Within the SCF framework, the contributions of polymers and solvent to the entropy and the chemical work can be calculated separately (see the Supplemental Material for technical details [56]). The results are shown in Fig. 4. The dominant terms are the polymer entropy and the chemical work associated with the polymers.

The polymer entropy decreases with increasing polydispersity $Đ$. This supports the packing hypothesis: Well-packed polymers fluctuate less and explore fewer conformations, which reduces their entropy. Interestingly, micelle formation in polydisperse systems does not lead to a reduction of interaction energy $U$ : Except in systems with fixed solvophobic block length, $\Delta U$ is positive. The picture changes in the canonical ensemble, where the number of polymers is fixed and one must consider the internal energy per polymer segment. This quantity is smaller in micelles than in the bulk (see Fig. 4, inset). Hence micelle formation is driven by a gain in energy per monomer but not necessarily by a gain in 

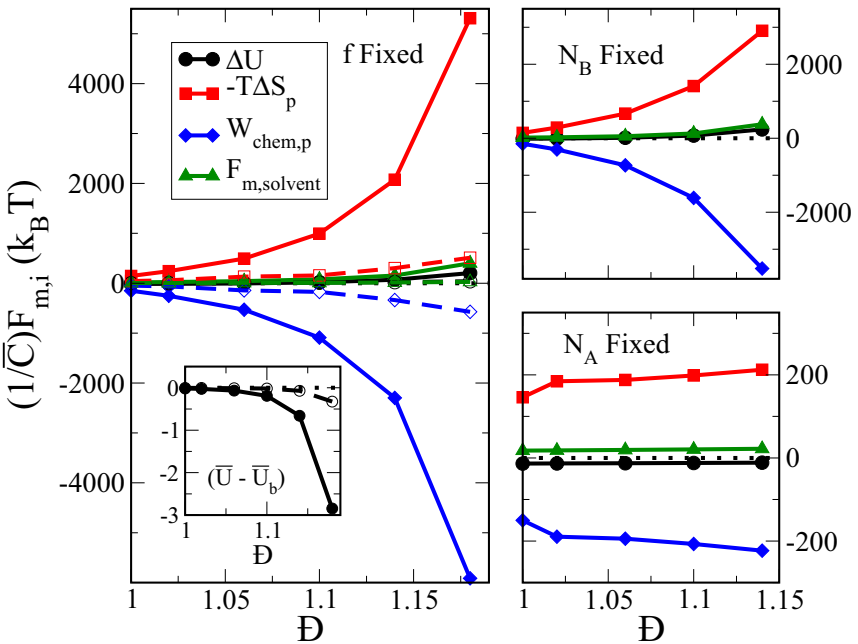

FIG. 4. Contributions to the micelle free energies from the interaction energy (black circles), the polymer entropy (red squares), the chemical work associated with the polymers (blue diamonds), and the sum of solvent entropy and solvent chemical work (green triangles) for the systems considered in this work. Full lines/full symbols refer to most probable micelles; dashed lines/open symbols to critical micelles. Inset shows the difference between the internal energy per polymer segment in the micelle system and in the bulk (in arbitrary units).

total energy in a grand canonical setup. The main negative contribution to $F_{m}$ favoring micelle formation is the chemical work associated with the polymers. It becomes stronger with increasing Đ. The chemical potential "pushes" polymers into the solution, and the system gains free energy if it can accommodate more polymers in the micelles. This again supports the packing hypothesis.

The proposed stabilizing mechanism is illustrated in Fig. 2 (cartoons). When forming spherical micelle cores from monodisperse solvophobic blocks, one necessarily creates frustration: Some blocks must stretch and some must compress to fill the space in the core. Therefore, the micelles offer little resistance to size variations. In contrast, polydisperse solvophobic blocks can optimize the packing inside the micelle and minimize the frustration, which makes them more stable.

\section{DISCUSSION}

In summary, we have investigated the effect of polymer length dispersity on self-assembled micelles in solutions of amphiphilic diblock copolymers. Our main results can be summarized as follows: (i) Consistent with previous studies [42-45], we find that the chain composition in micelles differs from that in solution-chains are longer on average. The reason is that in polydisperse systems, long polymers can segregate from solution and gain energy by forming micelles at lower cost of translational entropy. As a consequence, the size of the micelles increases compared to monodisperse systems, in agreement with experimental data [49]. (ii) With increasing polydispersity, the free energy barrier for micelle formation and dissolution increases, and (iii) the width of the size distribution of micelles decreases. Hence polymer polydispersity stabilizes micelles and reduces their size dispersity. A free energy analysis suggests that this phenomenon is driven by packing in the hydrophobic core, which is more efficient if the chains are polydisperse.

In the present work, we have considered a reference "bulk solution" where polymer segments are homogeneously distributed in the solution. In reality, the solvophobic blocks of individual chains may be collapsed $[63,64]$. This will affect the free energies of the reference state and the position of the $\mathrm{CMC}$ and also have an influence on the chain length distribution in the micelle. Unfortunately, studying these effects in a fully consistent manner is not possible in grand canonical SCF calculations, since the homogeneous bulk solution serves as outer boundary condition (see, e.g., Fig. 1). We plan to analyze this problem in more detail in the future.

We have considered moderate values of the polydispersity

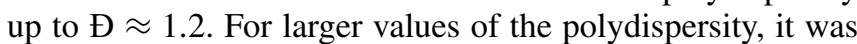
not possible to find a solution of the radial SCF equations, suggesting that spherical micelles may no longer be stable. Indeed, experiments suggest that large polydispersities may induce shape transitions and even morphological transitions [49]. This will be an interesting subject for future work.

We have considered systems close to the CMC, where micelles just begin to form, such that most copolymers are still in solution. Furthermore, we have assumed that micelles and micelle size distributions are fully equilibrated. This corresponds to an experimental situation where micelles are synthesized very slowly from a solution which does not change with time and provides an inexhaustible polymer reservoir. In reality, micelles consume polymers and the polymer composition changes during the process of micelle formation. Moreover, nanoparticles are not equilibrated. Their sizes, size distributions, and even morphologies depend on the parameters of the synthesis process [65-67]. Nevertheless, we believe that the insights from the present equilibrium considerations should also be relevant for real nonequilibrium processes and could provide useful guidance for experimental synthesis procedures. Roughly speaking, our study suggests that it may be easier to assemble well-defined polymeric nanoparticles with narrow size distribution from "bad" batches of polydisperse building blocks than from "good" batches of narrowly distributed building blocks, because the "bad" batches provide a range of different molecules which can be combined to optimize packing. This might be a general principle in solution self-assembly for nanoparticle synthesis.

\section{ACKNOWLEDGMENTS}

This research was partly supported by the German Science Foundation (DFG) via SFB 1066 (Grant No. 213555243, project Q1) and SFB TRR 146 (Grant No. 233630050, project C1). S.Q. acknowledges research support from the National Natural Science Foundation of China under the Grant No. NSFC-21873010. The simulations were carried out on the high performance computing center MOGON at JGU Mainz. 
[1] M. A. Bucaro, P. R. Kolodner, J. A. Taylor, A. Sidorenko, J. Aizenberg, and T. N. Krupenkin, Tunable liquid optics: Electrowetting-controlled liquid mirrors based on selfassembled janus tiles, Langmuir 25, 3876 (2009).

[2] A. Ethirajan and K. Landfester, Functional hybrid materials with polymer nanoparticles as templates, Chemistry 16, 9398 (2010).

[3] A. Nasir, A. Kausar, and A. Younus, A review on proparation, properties and applications of polymeric nanoparticle-based materials, Polym. Plast. Tech. Eng. 54, 325 (2015).

[4] K. Ulbrich, K. Holá, V. Šubr, A. Bakandritsos, J. Tuček, and R. Zbořil, Targeted drug delivery with polymers and magnetic nanoparticles: Covalent and noncovalent approaches, release control, and clinical studies, Chem. Rev. 116, 5338 (2016).

[5] M. W. Tibbitt, J. E. Dahlman, and R. Langer, Emerging frontiers in drug delivery, J. Am. Chem. Soc. 138, 704 (2016).

[6] S. Förster and T. Plantenberg, From self-organizing polymers to nanohybrid and biomaterials, Angew. Chemie Intnl. Ed. 41, 688 (2002).

[7] J. Rodriguez-Hernandez, F. Checot, Y. Gnanou, and S. Lecommandoux, Toward 'smart' nano-objects by self-assembly of block copolymers in solution, Prog. Polym. Sci. 30, 691 (2005).

[8] F. A. M. Leermakers, C. M. Wijmans, and G. J. Fleer, On the structure of polymeric micelles: Self-consistent-field theory and universal properties for volume fraction profiles, Macromolecules 28, 3434 (1995).

[9] S. E. Webber, Polymer micelles: an example of self-assembling polymers, J. Phys. Chem. B 102, 2618 (1998).

[10] G. Riess, Micellization of block copolymers, Prog. Polym. Sci. 28, 1107 (2003).

[11] J. G. J. L. Lebouille, L. F. W. Vleugels, A. A. Dias, F. A. M. Leermakers, M. A. Cohen Stuart, and R. Tuinier, Controlled block copolymer micelle formation for encapsulation of hydrophobic ingredients, Eur. Phys. J. E 36, 107 (2013).

[12] S.-T. Feng, J. Li, Y. Luo, T. Yin, H. Cai, Y. Wang, Z. Dong, X. Shuai, and Z.-P. Li, ph-sensitive nanomicelles for controlled and efficient drug delivery to human colorectal carcinoma lovo cells, PLOS ONE 9, e100732 (2014).

[13] M. Nakayama and T. Okano, Intelligent thermoresponsive polymeric micelles for targeted drug delivery, J. Drug Delivery Sci. Technol. 16, 35 (2006).

[14] K. Kataoka, A. Harada, and Y. Nagasaki, Block copolymer micelles for drug delivery: Design, characterization and biological significance, Adv. Drug Delivery Rev. 47, 113 (2001).

[15] H. Cabral, Y. Matsumoto, K. Mizuno, Q. Chen, M. Murakami, M. Kimura, Y. Terada, M. R. Kano, K. Miyazono, M. Uesaka, N. Nishiyama, and K. Kataoka, Accumulation of sub-100 nm polymeric micelles in poorly permeable tumours depends on size, Nat. Nanotechnol. 6, 815 (2011).

[16] T. Chang, M. S. Lord, B. Bergmann, A. Macmillan, and M. H. Stenzel, Size effects of self-assembled block copolymer spherical micelles and vesicles on cellular uptake in human colon carcinoma cells, J. Mater. Chem. B 2, 2883 (2014).

[17] K.-I. Seno, S. Kanaoka, and S. Aoshima, Thermosensitive diblock copolymers with designed molecular weight distribution: Synthesis by continuous living cationic polymerization and micellization behavior, J. Polymer Sci. A 46, 2212 (2007).
[18] N. A. Lynd, A. J. Meuler, and M. A. Hillmyer, Polydispersity and block copolymer self-assembly, Prog. Polym. Sci. 33, 875 (2008).

[19] K. E. B. Doncom, L. D. Blackman, D. B. Wright, M. I. Gibson, and R. K. O'Reilly, Dispersity effects in polymer selfassemblies: a matter of hierarchical control, Chem. Soc. Rev. 46, 4119 (2017).

[20] P. H. Nelson, G. C. Rutledge, and T. A. Hatton, On the size and shape of self-assembled micelles, J. Chem. Phys. 107, 10777 (1997).

[21] J. M. Widin, A. K. Schmitt, A. L. Schmitt, K. Im, and M. K. Mahanthappa, Unexpected consequences of block polydispersity on the self-assembly of aba triblock copolymers, J. Am. Chem. Soc. 134, 3834 (2012)

[22] S. Qi, L. I. Klushin, A. M. Skvortsov, and F. Schmid, Polydisperse polymer brushes: Internal structure, critical behavior and interaction with flow, Macromolecules 49, 9655 (2016).

[23] D. Romeis and J.-U. Sommer, Binary and bidisperse polymer brushes: Coexisting surface states, ACS Appl. Mater. Interfaces 7, 12496 (2015).

[24] A. M. Skvortsov, L. I. Klushin, and A. A. Gorbunov, Long and short chains in a polymeric brush: A conformational transition, Macromolecules 30, 1818 (1997).

[25] H. Merlitz, G.-L. He, C.-V. Wu, and J.-U. Sommer, Surface instabilities of monodisperse and densely grafted polymer brushes, Macromolecules 41, 5070 (2008).

[26] D. Romeis and J.-U. Sommer, Conformational switching of modified guest chains in polymer brushes, J. Chem. Phys. 139, 044910 (2013).

[27] D. Nguyen, X.-F. Zhong, C. E. Williams, and A. Eisenberg, Effect of ionic chain polydispersity on the size of spherical ionic microdomains in diblock ionomers, Macromolecules 27, 5173 (1994).

[28] Y. Matsushita, A. Noro, M. Iinuma, J. Suzuki, H. Ohtani, and A. Takano, Effect of composition distribution on microphaseseparated structure from diblock copolymers, Macromolecules 36, 8074 (2003).

[29] S. W. Sides and G. H. Fredrickson, Continuous polydispersity in a self-consistent field theory for diblock copolymers, J. Chem. Phys. 121, 4974 (2004).

[30] A. Noro, D. Cho, A. Takano, and Y. Matsushita, Effect of molecular weight distribution on microphase-separated structures from block copolymers, Macromolecules 38, 4371 (2005).

[31] J. Wang, Z.-G. Wang, and Y. Yang, Nature of disordered micelles in sphere-forming block copolymer melts, Macromolecules 38, 1979 (2005).

[32] A.-V. Ruzette, S. Tencé-Girault, L. Leibler, F. Chauvin, D. Bertin, O. Guerret, and P. Gérard, Molecular disorder and mesoscopic order in polydisperse acrylic block copolymers prepared by controlled radical polymerization, Macromolecules 39, 5804 (2006).

[33] N. Torikai, A. Noro, M. Okuda, F. Odamaki, D. Kawaguchi, A. Takano, and Y. Matsushita, Neutron reflection studies on lamellar microphase-separated structures of two-component block copolymers with composition distribution, Physica B 385-386, 709 (2006). 
[34] N. A. Lynd and M. A. Hillmyer, Influence of polydispersity on the self-assembly of diblock copolymers, Macromolecules $\mathbf{3 8}$, 8803 (2005).

[35] N. A. Lynd and M. A. Hillmyer, Effects of polydispersity on the order-disorder transition in block copolymer melts, Macromolecules 40, 8050 (2007).

[36] N. A. Lynd, B. D. Hamilton, and M. A. Hillmyer, The role of polydispersity in the lamellar mesophase of model diblock copolymers, J. Polym. Sci., Part B: Polym. Phys. 45, 3386 (2007).

[37] S. Park, D. Y. Ryu, J. K. Kim, M. Ree, and T. Chang, Property of diblock copolymer having extremely narrow molecular weight distribution, Polymer 49, 2170 (2008).

[38] M. W. Matsen, Effect of large degrees of polydispersity on strongly segregated block copolymers, Eur. Phys. J. E 21, 199 (2006).

[39] M. W. Matsen, Polydispersity-Induced Macrophase Separation in Diblock Copolymer Melts, Phys. Rev. Lett. 99, 148304 (2007).

[40] D. M. Cooke and A.-C. Shi, Effects of polydispersity on phase behavior of diblock copolymers, Macromolecules 39, 6661 (2006).

[41] B. Oschmann, J. Lawrence, M. W. Schulze, J. M. Ren, A. Anastasak, Y. Luo, M. D. Nothling, C. W. Pester, K. T. Delaney, L. A. Connal, A. J. Mcgrath, P. G. Clark, C. M. Bates, and C. J. Hawker, Effects of tailored dispersity on the self-assembly of dimethylsiloxane-methyl methacrylate block co-oligomers, ACS Macro Lett 6, 668 (2017).

[42] Z. Gao and A. Eisenberg, A model of micellization for block copolymers in solutions, Macromolecules 26, 7353 (1993).

[43] P. Linse, Micellization of poly(ethylene oxide)-poly(propylene oxide) block copolymers in aqueous solution: Effect of polymer polydispersity, Macromolecules 27, 6404 (1994).

[44] K. Khougaz, Z. Gao, and A. Eisenberg, Macromolecules 27, 6341 (1994).

[45] S. Hvidt, C. Trandum, and W. Batsberg, Effects of poloxamer polydispersity on micellization in water, J. Coll. Interf. Sci. 250, 243 (2002).

[46] O. Terreau, L. Luo, and A. Eisenberg, Effect of poly(acrylic acid) block length distribution on polystyrene- $b$-poly(acrylic acid) aggregates in solution. 1. vesicles, Langmuir 19, 5601 (2003).

[47] Y. Jiang, T. Chen, F. Ye, H. Liang, and A.-C. Shi, Effect of polydispersity on the formation of vesicles from amphiphilic diblock copolymers, Macromolecules 38, 6710 (2005).

[48] F. Li, S. Prevost, R. Schweins, A. T. M. Marcelis, F. A. M. Leermakers, M. A. Cohen Stuart, and E. J. R. Sudhölter, Small monodisperse unilamellar vesicles from binary copolymer mixtures, Soft Matter 5, 4169 (2009).

[49] A. L. Schmitt, M. H. Repollet-Pedrosa, and M. K. Mahanthappa, Polydispersity-driven block copolymer amphiphile self-assembly into prolate-spheroid micelles, ACS Macro Lett. 1, 300 (2012).
[50] G. H. Fredrickson and S. W. Sides, Theory of polydisperse inhomogeneous polymers, Macromolecules 36, 5415 (2003).

[51] M. Doi and S. F. Edwards, The Theory of Polymer Dynamics (Oxford University Press, Oxford, 1986).

[52] G. H. Fredrickson, The Equilibrium Theory of Inhomogeneous Polymers (Oxford University Press, Oxford, 2006).

[53] X. He and F. Schmid, Dynamics of spontaneous vesicle formation in dilute solutions of amphiphilic diblock copolymers, Macromolecules 39, 2654 (2006).

[54] E. Helfand, Theory of inhomogeneous polymers: Fundamentals of the gaussian random-walk model, J. Chem. Phys. 62, 999 (1975).

[55] F. Schmid, Self-consistent field theories for complex fluids, J. Phys.: Condens. Matter 10, 8105 (1998).

[56] See Supplemental Material at http://link.aps.org/supplemental/ 10.1103/PhysRevMaterials.3.026002 for detailed description of the model, SCF equations, and the thermodynamic analysis.

[57] F. Schmid, Theory and simulation of multiphase polymer systems, in Handbook of Multiphase Polymer Systems (WileyBlackwell, West Sussex, 2011), Chap. 3, pp. 31-80.

[58] S. Qi and D. Yan, Nucleation in polydisperse polymer mixtures, J. Chem. Phys. 129, 204902 (2008).

[59] J. Glaser, P. Medapuram, T. M. Beardsley, M. W. Matsen, and D. C. Morse, Universality of Block Copolymer Melts, Phys. Rev. Lett. 113, 068302 (2014).

[60] F. S. Bates, M. F. Schulz, A. K. Khandpur, S. Förster, J. H. Rosedale, K. Almdal, and K. Mortensen, Fluctuations, conformational asymmetry and block copolymer phase behaviour, Faraday Discuss. 98, 7 (1994).

[61] F. S. Bates, J. H. Rosedale, and G. H. Fredrickson, Fluctuation effects in a symmetric diblock copolymer near the orderdisorder transition, J. Chem. Phys. 92, 6255 (1990).

[62] F. S. Bates, J. H. Rosedale, G. H. Fredrickson, and C. J. Glinka, Fluctuation-Induced First-Order Transition of an Isotropic System to a Periodic State, Phys. Rev. Lett. 61, 2229 (1988).

[63] J. Wang, K. Guo, L. An, M. Müller, and Z.-G. Wang, Micelles of coil-comb block copolymers in selective solvents: Competition of length scales, Macromolecules 43, 2037 (2010).

[64] R. Wang and Z.-G. Wang, Theory of polymers in poor solvent: Phase equilibrium and nucleation behavior, Macromolecules 45, 6266 (2012).

[65] R. Bleul, R. Thiermann, and M. Maskos, Techniques to control polymersome size, Macromolecules 48, 7396 (2015).

[66] A. Nikoubashman, V. E. Lee, C. Sosa, R. K. Prud'homme, R. D. Priestley, and A. Z. Panagiotopoulos, Directed Assembly of Soft Colloids through Rapid Solvent Exchange, ACS Nano 10, 1425 (2016).

[67] S. Kessler, K. Drese, and F. Schmid, Simulating copolymeric nanoparticle assembly in the co-solvent method: How mixing rates control final particle sizes and morphologies, Polymer 126C, 9 (2017). 